\title{
Convergence of a Vortex In Cell Method for the Two-Dimensional Euler Equations
}

\author{
By G. H. Cottet
}

\begin{abstract}
We describe a Vortex In Cell method in which the assignment function used to compute vorticity values at the grid points from particles is twice differentiable, while the velocity need only be continuous. We prove an error estimate for the velocity in terms of the meshsize, the interparticle distance and the size of the computational domain.
\end{abstract}

1. Introduction. Consider the Euler equations in the whole plane $\mathbf{R}^{2}$, which can be written in terms of vorticity $\omega$ and stream function $\psi$ in the following way:

$$
\text { (E) }\left\{\begin{array}{l}
\frac{\partial \omega}{\partial t}+(\overrightarrow{\operatorname{curl}} \psi \cdot \vec{\nabla}) \omega=0, \\
-\Delta \psi=\omega, \\
|\vec{\nabla} \psi| \rightarrow 0 \text { when }|x| \rightarrow+\infty, \\
\omega(x, 0)=\omega_{0}(x) .
\end{array}\right.
$$

Recently, vortex methods have been introduced to approximate equations (E). The first proof of convergence has been given by Hald [6], then generalized by Beale and Majda [1] and Cottet [3]. In such methods the basic idea is

(1) to discretize the initial vorticity by delta functions;

(2) to calculate the velocity field by using a regularized Green kernel;

(3) to transport the vorticity by the velocity field.

It is natural to think of a finite element method rather than an integral one in step (2). The approximation then results in coupling a particle approximation (to solve E.1, E.4) and a finite-difference method on a fixed grid (to solve E.2, E.3). Such methods, called Vortex In Cell methods, are extensively used in practice. In particular, they are preferred to methods using explicit kernel calculations because in many situations they lead to a smaller computational cost. Moreover, such methods have the particular feature of naturally taking into account more general boundary conditions, for instance periodic boundary conditions.

However, for technical simplicity, we consider here the approximation in the whole plane.

An outline of the paper is as follows. In Section 2 a VIC method with TSC assignment function (in the terminology of [7]) is described using either finite element or finite-difference methodologies. Section 3 is devoted to the properties of

Received March 20, 1985; revised January 12, 1987.

1980 Mathematics Subject Classification (1985 Revision). Primary 65M15; Secondary 76D05. 
the resulting Poisson's solver. The proof of convergence is then given in Section 4; it is strongly based on the results given in [3] for vortex methods with nonsmooth cutoff functions. Finally, error estimates are discussed in Section 5, together with possible improvements of the method. For a different analysis of VIC methods we refer to the recent work [4], and to [5] in the context of plasma-physics.

2. Description of the Method. Throughout this paper we shall assume, for technical simplicity, that the initial vorticity $\omega_{0}$ has compact support.

Given a parameter $\Delta x>0$, consider a uniform partition of supp $\omega_{0}$ by rectangles $B_{k}$,

$$
B_{k}=\left\{x \in \mathbf{R}^{2} ;\left(k_{i}-\frac{1}{2}\right) \Delta x \leqslant x_{i} \leqslant\left(k_{i}+\frac{1}{2}\right) \Delta x\right\}, \quad k=\left(k_{1}, k_{2}\right) .
$$

Denoting by $x_{k}=\left(k_{1} \Delta x, k_{2} \Delta x\right)$ the center of $B_{k}$, we define the following approximation of $\omega_{0}$ :

$$
\omega_{0}^{h}(x)=\sum_{k} \alpha_{k} \delta\left(x-x_{k}\right), \text { where } \alpha_{k}=\Delta x^{2} \omega_{0}\left(x_{k}\right) .
$$

Here, and everywhere in the sequel, we write $\sum_{k}$ to denote the finite summation $\sum_{|k| \leqslant N}, N$ being some ad hoc constant.

Now, given an approximation $\vec{u}^{h}$ of $\vec{u}=\overrightarrow{\operatorname{rot}} \psi$, let $\left(X_{k}^{h}\right)_{k}$ be the solution of the system of ordinary differential equations

$$
\left\{\begin{array}{l}
d X_{k}^{h} / d s=\vec{u}\left(X_{k}^{h}(s), s\right), \\
X_{k}^{h}(0)=x_{k}, \quad k \in \mathbf{Z}^{2},|k| \leqslant N .
\end{array}\right.
$$

The measure solution of the system

$$
\left\{\begin{array}{l}
\partial \omega^{h} / \partial t+\left(\vec{u}^{h} \cdot \vec{\nabla}\right) \omega^{h}=0 \\
\omega^{h}(\cdot, 0)=\omega_{0}^{h}
\end{array}\right.
$$

is then given by

$$
\omega^{h}(x, t)=\sum_{k} \alpha_{k} \delta\left(x-X_{k}^{h}(t)\right) .
$$

To specify our approximation of the problem (E), we now have to couple $\vec{u}^{h}$ and $\omega^{h}$ by solving some analogue of (1.2), (1.3).

For this purpose, and in order to work on a bounded domain, we introduce for $R>0$ the square $\Omega_{R}=(-R,+R)^{2}$, with boundary $\Gamma_{R}$. Then we consider the following Neumann problem, which is denoted by $P_{R}(\omega)$,

$$
\left\{\begin{array}{l}
-\Delta \psi=\omega \quad \text { in } \Omega_{R}, \\
\partial \psi / \partial n=C_{R}(\omega) \text { on } \Gamma_{R},
\end{array}\right.
$$

where the constant $C_{R}(\omega)$ is given by

$$
C_{R}(\omega)=-\frac{1}{8 R} \int_{\Omega_{R}} \omega(x) d x
$$

so that $P_{R}(\omega)$ is a well-posed problem.

Together with $P_{R}(\omega)$ we may introduce the linear mapping $\mathscr{S}$ such that, if $\psi$ is the solution of $P_{R}(\omega)$ (unique up to an additive constant), $\mathscr{S}(\omega)$ is the following vector-valued function:

$$
\mathscr{S}(\omega)= \begin{cases}\overrightarrow{\operatorname{rot}} \psi & \text { in } \Omega_{R}, \\ 0 & \text { elsewhere. }\end{cases}
$$


We now define a $P_{1}$-finite element approximation of $P_{R}(\omega)$ in this way: Given $\varepsilon>0$ such that $R / \varepsilon \in \mathbf{N}$, denote by $x_{i j}$ the points

$$
x_{i j}=(i \varepsilon, j \varepsilon), \quad(i, j) \in \mathbf{Z}^{2},
$$

and by $\mathscr{T}_{h}$ the triangulation of $\Omega_{R}$ whose vertices are the $x_{i j}$ which lie in $\Omega_{R}$ and whose sides are parallel to the axes and the first diagonal. Finally, define

$$
\mathscr{M}_{h}=\left\{\psi \in C\left(\bar{\Omega}_{R}\right), \psi_{\mid T} \text { is linear for any } T \text { in } \mathscr{T}_{h}\right\} .
$$

$\mathscr{M}_{h}$ is provided with its usual basis $\left\{w_{\varepsilon}\left(x-x_{i j}\right)\right\}_{(i, j) \in \mathbf{Z}^{2}}$. Then consider the approximate problem $P^{h}(\omega)$ :

$$
\left\{\begin{array}{l}
\text { Find } \psi^{h} \in \mathscr{M}_{h} \text { such that } \\
\int_{\Omega_{R}} \nabla \psi^{h} \cdot \nabla \varphi^{h} d x=\int_{\Omega_{R}} \omega \cdot \varphi^{h} d x+\int_{\Gamma_{R}} C_{R}(\omega) \varphi^{h} d \gamma \text { for all } \varphi^{h} \text { in } \mathscr{M}_{h} .
\end{array}\right.
$$

Derivatives of $\psi^{h}$ are piecewise constant; in order to handle continuous quantities, we first introduce in a classical way the differential quotients for a continuous function $\psi$,

$$
\begin{cases}\left(\partial_{1} \psi\right)_{i, j}=\frac{1}{2 \varepsilon}\left(\psi_{i+1, j}-\psi_{i-1, j}\right), & \left(\partial_{2} \psi\right)_{i, j}=\frac{1}{2 \varepsilon}\left(\psi_{i, j+1}-\psi_{i, j-1}\right), \\ \left(\partial_{1} \psi\right)_{i+1 / 2, j}=\frac{1}{\varepsilon}\left(\psi_{i+1, j}-\psi_{i, j}\right), & \left(\partial_{2} \psi\right)_{i, j+1 / 2}=\frac{1}{\varepsilon}\left(\psi_{i, j+1}-\psi_{i, j}\right),\end{cases}
$$

where $\psi_{i, j}=\psi\left(x_{i j}\right)$, and we define the mapping $\mathscr{S}^{h}$ :

$$
\mathscr{S}^{h}(\omega)_{i, j}= \begin{cases}\left(\left(\partial_{2} \psi^{n}\right)_{i, j},-\left(\partial_{1} \psi^{h}\right)_{i, j}\right) & \text { if } x_{i j} \in \bar{\Omega}_{3 R / 4}, \\ 0 & \text { if not, }\end{cases}
$$

where $\psi^{h}$ is the solution of $P_{h}(\omega)$. We then define

$$
\mathscr{S}^{h}(\omega)(x)=\sum_{i, j} \mathscr{S}^{h}(\omega)_{i, j} w_{\varepsilon}\left(x-x_{i j}\right), \quad x \in \mathbf{R}^{2} .
$$

A natural coupling of $\vec{u}^{h}$ and $\omega^{h}$ would be to set $\vec{u}^{h}=\mathscr{S}^{h}\left(\omega^{h}\right)$. In fact, in order to ensure stability, we have first to regularize $\omega^{h}$.

Let $\chi$ be the characteristic function of $\left(-\frac{1}{2},+\frac{1}{2}\right)^{2}$ and

$$
\chi_{\varepsilon}: x \mapsto \varepsilon^{-2} \chi(x / \varepsilon) \text {. }
$$

We set

$$
\tilde{\omega}^{h}=\omega^{h} * \chi_{\varepsilon}
$$

and

$$
\vec{u}^{h}=\mathscr{S}^{h}\left(\tilde{\omega}^{h}\right) .
$$

Our approximate problem finally results in (2.1), (2.3), (2.11). Observe that the parameter $h$ of the approximation takes into account all of $\Delta x, \varepsilon$ and $R$.

We shall first have to prove the following existence and uniqueness result.

THEOREM 1. For all $T>0$, there exist a unique measure $\omega^{h}$ and a unique continuous function $\vec{u}^{h}$, solutions of the equations $(2.1),(2.3),(2.11)$. 
Then our main result is the following convergence theorem.

THEOREM 2. Let $T>0$. Suppose that $\omega_{0}$ has compact support and that the solution $\vec{u}$ of the Euler equations is sufficiently smooth.

Given $p \in(2,+\infty), s>1$, there exist constants $C_{i}$ and $\varepsilon_{0}$ such that under the assumptions

$$
\varepsilon \leqslant \varepsilon_{0}, \quad C_{1} \varepsilon^{s} \leqslant \Delta x \leqslant C_{2} \varepsilon^{s}, \quad R \geqslant C_{3} \varepsilon^{s /(2 / p-1)}
$$

we get for all $t \in[0, T]$

$$
\left\|\left(\vec{u}-\vec{u}^{h}\right)(\cdot, t)\right\|_{\left(L^{p}\left(\mathbf{R}^{2}\right)\right)^{2}} \leqslant C\left\{\varepsilon^{2}+\frac{\Delta x^{2}}{\varepsilon}+R^{(2 / p)-1}\right\} .
$$

Before giving the proof of these results, we state a version of our method based on a finite-difference formulation of $\mathscr{S}^{h}$, which is more suitable for a "Particle in Cell" interpretation.

To get this alternative formulation, the first step is to assign values to $\omega^{h}$ on the grid points $x_{i j}$, when $\omega^{h}$ is not a continuous function. This is done by setting

$$
\omega_{i, j}^{h}=\frac{1}{\varepsilon^{2}}\left[\omega^{h} *\left(\chi_{\varepsilon} * w_{\varepsilon}\right)\right]\left(x_{i j}\right)
$$

or, in view of (2.4),

$$
\omega_{i, j}^{h}=\frac{1}{\varepsilon^{2}} \sum_{k \in \mathbf{Z}^{2}} \alpha_{k}\left(\chi_{\varepsilon} * w_{\varepsilon}\right)\left(x_{i j}-X_{k}^{h}(t)\right) .
$$

Then we are looking for a grid function $\left(\psi_{i, j}^{h}\right)_{(i, j) \in \mathbf{Z}^{2}}$ such that

$$
\begin{gathered}
\left(\Delta^{\varepsilon} \psi^{h}\right)_{i, j}=\omega_{i, j}^{h} \quad \text { if }|i \varepsilon|<R,|j \varepsilon|<R, \\
\frac{1}{\varepsilon^{2}}\left[-\frac{1}{2} \psi_{i, j-1}^{h}-\frac{1}{2} \psi_{i, j+1}^{h}-\psi_{i-1, j}^{h}+2 \psi_{i, j}^{h}\right]=\omega_{i, j}^{h}+\frac{C_{R}\left(\omega^{h}\right)}{\varepsilon} \\
\text { if } i \varepsilon=R \text { and }|j \varepsilon|<R, \\
\frac{1}{\varepsilon^{2}}\left[-\frac{1}{2} \psi_{i, j-1}^{h}-\frac{1}{2} \psi_{i-1, j}^{h}+\psi_{i, j}^{h}\right]=\omega_{i, j}^{h}+\frac{C_{R}\left(\omega^{h}\right)}{\varepsilon} \\
\text { if } i \varepsilon=R, j \varepsilon=R,
\end{gathered}
$$

where $\Delta^{\varepsilon}$ denotes the usual five-point approximation of the Laplace operator on the $x_{i j}$ grid, the other sides and vertices of $\Omega_{R}$ being treated in a similar fashion. Next, we set

$$
\vec{u}_{i, j}^{h}= \begin{cases}\left(\left(\partial_{2} \psi^{h}\right)_{i, j},-\left(\partial_{1} \psi^{h}\right)_{i, j}\right) & \text { if } x_{i j} \in \bar{\Omega}_{3 R / 4}, \\ 0 & \text { if not, }\end{cases}
$$

and

$$
\vec{u}^{h}(x)=\sum_{i, j} \vec{u}_{i, j}^{h} w_{\varepsilon}\left(x-x_{i j}\right), \quad x \in \mathbf{R}^{2}
$$

We claim that the formulations (2.10), (2.11) and (2.14)-(2.16), (2.17), respectively, are equivalent. To see this, write $\psi^{h}=\sum_{i, j} \psi_{i, j}^{h} w_{\varepsilon}\left(x-x_{i j}\right)$ and choose $\varphi^{h}=$ $w_{\varepsilon}\left(x-x_{i_{0} j_{0}}\right)$ in (2.7). Then we obtain (2.14), (2.15) or (2.16), depending on whether $x_{i_{0} j_{0}}$ is located in the interior on $\Omega_{R}$, in $\Gamma_{R}$, or at a corner of $\Omega_{R}$, respectively. 
From this new formulation of $\mathscr{S}^{h}$ it is now possible to describe our method in terms of Vortex In Cell terminology.

Equation (2.12) defines a vorticity assignment to the grid points, i.e., it shows how to compute the vorticity at $x_{i j}$ in terms of the vortices $X_{k}^{h}$. Only those vortices which ensure $\left(\chi_{\varepsilon} * w_{\varepsilon}\right)\left(x_{i j}-X_{k}^{h}(t)\right) \neq 0$ give a contribution at $x_{i j}$. These vortices are clearly located in the shaded area of the following drawing

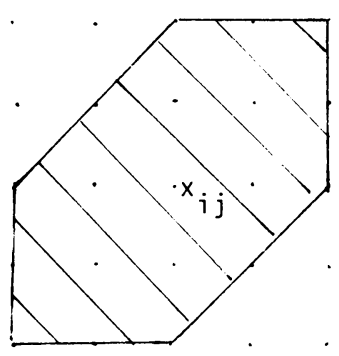

As a consequence, the vorticity carried by a particle is shared among the nine nearest grid points. This observation, together with the $W^{2, \infty}$ regularity of the assignment function $\chi_{\varepsilon} * w_{\varepsilon}$, enables us to speak of a TSC assignment scheme (see [7]).

On the other hand, (2.11) defines $\vec{u}^{h}$ as a piecewise $P_{1}$ function, interpolated from the values $\vec{u}_{i j}^{h}$ computed on four grid points. Our method may thus be viewed as a mixed PIC-TSC method.

3. Preliminary Results Concerning the Mapping $\mathscr{S}^{h}$. In this section our purpose is to derive properties of stability, decay at infinity and consistency for $\mathscr{S}^{h}$. The parameter $h$ will therefore only take into account $\varepsilon$ and $R$, and we shall set $h=(\varepsilon, R)$.

Concerning stability and consistency, we first prove the following result.

LEMMA 3.1. Let $\omega$ be in $L^{\infty}\left(\mathbf{R}^{2}\right)$ and $\psi$ be the solution (unique up to a constant) of $P(\omega)$. There exist constants $C_{p}$ independent of $h$ such that

$$
\begin{array}{cc}
\left\|\mathscr{S}^{h}(\omega)\right\|_{L^{p}\left(\Omega_{R}\right)} \leqslant C_{p}\left\{|\psi|_{1, p, \Omega_{R}}+\frac{1}{R}\|\psi\|_{L^{p}\left(\Omega_{R}\right)}\right\}, & p \in[2,+\infty], \\
\left\|\mathscr{S}^{h}(\omega)-\overrightarrow{\operatorname{rot}} \psi\right\|_{L^{p}\left(\Omega_{3 R / 4}\right)} \leqslant C_{p} \varepsilon\|\psi\|_{W^{2, p}\left(\Omega_{R}\right)}, & p \in[2,+\infty] .
\end{array}
$$

Proof. It is convenient to work on a reference domain $\hat{\Omega}=\Omega_{1}$. Setting

$$
\begin{gathered}
\hat{\omega}(\hat{x})=\omega(R \hat{x}), \quad \hat{x} \in \hat{\Omega}, \\
\hat{C}=\frac{1}{R} C_{R}(\omega), \quad \hat{h}=\left(\frac{\varepsilon}{R}, 1\right),
\end{gathered}
$$

it is readily seen that if $\psi$ and $\psi^{h}$ are solutions of $P_{R}(\omega)$ and $P^{h}(\omega)$, then $\hat{\psi}$ and $\hat{\psi}^{h}$ defined by

$$
\hat{\psi}(\hat{x})=\frac{1}{R^{2}} \psi(R \hat{x}), \quad \hat{\psi}^{h}(\hat{x})=\frac{1}{R^{2}} \psi^{h}(R \hat{x})
$$


are solutions of $P_{1}(\hat{\omega})$ and $P^{\hat{h}}(\hat{\omega})$. Hence, techniques used by Rannacher and Scott in [9] allow us to derive the following estimates:

$$
\left|\hat{\psi}^{h}\right|_{1, P, \hat{\Omega}} \leqslant C_{p}\|\hat{\psi}\|_{1, p, \hat{\Omega}}, \quad\left|\hat{\psi}-\hat{\psi}^{h}\right|_{1, p, \hat{\Omega}} \leqslant C_{p} \frac{\varepsilon}{R}\|\hat{\psi}\|_{2, p, \hat{\Omega}}
$$

To come back to $\Omega_{R}$ and obtain (3.1) and (3.2), it remains to use the following obvious identities:

$$
\left\|D^{l} \hat{\psi}\right\|_{L^{p}(\hat{\Omega})}=R^{l-2-(2 / p)}\left\|D^{l} \psi\right\|_{L^{p}\left(\Omega_{R}\right)}, \quad l \in \mathbf{N} .
$$

In the next lemma we summarize some classical results about the operators $\Delta$ and $\Delta^{\varepsilon}$ which will be extensively used in the sequel. For $R>0$, we denote by $\Omega_{R}^{\prime}$ (resp. $\Omega_{R}^{\prime \prime}$ ) the square $\Omega_{3 R / 4}$ (resp. $\Omega_{R / 2}$ ) and by $\Gamma_{R}^{\prime}$ (resp. $\Gamma_{R}^{\prime \prime}$ ) its boundary.

LEMMA 3.2. Let $\psi\left(\right.$ resp. $\psi^{h}$ ) be a continuous function (resp. a grid function on the grid $x_{i j}$ ) in $\bar{\Omega}_{R}$. Let $\partial_{l} \psi^{h}, l=1,2$, be the differential quotients defined by (2.8). Assume that $\Delta \psi=\Delta \psi^{h}=0$ in $\Omega_{R}$. Then we have

(i) $\|\psi\|_{L^{\infty}\left(\Omega_{R}\right)} \leqslant\|\psi\|_{L^{\infty}\left(\Gamma_{R}\right)}$,

(ii) $\operatorname{Max}_{x_{i j} \in \Omega_{R}}\left|\psi_{i, j}^{h}\right| \leqslant \operatorname{Max}_{x_{i j} \in \Gamma_{R}}\left|\psi_{i, j}^{h}\right|$,

(iii) $\operatorname{Max}_{x_{i j} \in \Omega_{R}^{\prime}}\left|\left(\partial, \psi^{h}\right)_{i, j}\right| \leqslant(C / R) \operatorname{Max}_{x_{i j} \in \Omega_{R}}\left|\psi_{i, j}^{h}\right|, l=1,2$.

Proof. Assertions (i) and (ii) are direct consequences of the continuous and the discrete maximum principles for $\Delta$ (see [2]). Assertion (iii) results from discrete interior Schauder estimates (see [11]). In fact, we first use the transform (3.3); this enables us to apply Schauder estimates in $\left(-\frac{3}{4},+\frac{3}{4}\right)^{2}$ which is strictly included in $\hat{\Omega}$. Coming back to $\Omega_{R}^{\prime}$ and $\Omega_{R}$, and using (3.4), provides us with the desired estimates.

As a first consequence of the estimates just given, we obtain the following lemma which gives the asymptotic behavior of $\mathscr{S}^{h}(\omega)$ when $|x|$ goes to infinity. It will be seen later that this result enables us to establish stability and consistency for the scheme, but it is interesting in its own right since it proves the uniform, with respect to $h$ and $\omega$, decay of $\mathscr{S}^{h}(\omega)$.

We introduce the following notation: If $\omega$ has compact support, we denote by $\rho(\omega)$ the real number

$$
\rho(\omega)=\operatorname{Max}\{|x|, \omega(x) \neq 0\}
$$

We can now state

LEMMA 3.3. Let $\omega$ be in $L^{1}\left(\mathbf{R}^{2}\right)$ with compact support. There exist constants $R_{0}$ and $C$, which only depend on $\|\omega\|_{L^{1}\left(\mathbf{R}^{2}\right)}$ and $\rho(\omega)$, such that if $R \geqslant R_{0}$ and $\varepsilon<1$ then

$$
\left|\mathscr{S}^{h}(\omega)(x)\right| \leqslant \frac{C}{|x|} \text { if }|x| \geqslant R_{0} .
$$

Proof. We shall make use of the existence of a fundamental solution for $\Delta^{\varepsilon}$. More precisely, we know that there exists (cf. [11] for instance) a grid function $g$, defined on the whole space $\mathbf{R}^{2}$, such that

$$
\left(-\Delta^{\varepsilon} g\right)_{i, j}=\varepsilon^{-2} \delta_{i, 0} \cdot \delta_{j, 0} \quad(\delta \text { is the Kronecker symbol }) ;
$$


moreover, $g$ verifies

$$
\left|\left(\partial_{l} g\right)_{i, j}\right| \leqslant \frac{C}{\varepsilon+\left|x_{i j}\right|}, \quad l=1,2 .
$$

Define the grid function $\psi_{0}^{h}$ by

$$
\psi_{0}^{h}\left(x_{i j}\right)=\sum_{k, l} \varepsilon^{2} \omega_{k, l} g\left(x_{i j}-x_{k l}\right)
$$

and set $R_{0}=\rho(\omega)+1$. Then one easily derives the following result:

$$
\begin{gathered}
\left(\Delta \psi_{0}^{h}\right)_{i, j}=\omega_{i, j}, \\
\left|\left(\partial_{m} \psi_{0}^{h}\right)_{i, j}\right| \leqslant \frac{C}{\left|x_{i j}\right|}\left(\sum_{k, l}\left|\omega_{k, l}\right|\right), \quad m=1,2 ;\left|x_{i j}\right| \geqslant 2 R_{0} .
\end{gathered}
$$

Hence, if $\psi^{h}$ is a solution of $P_{h}(\omega)$, one gets $\Delta^{\varepsilon}\left(\psi_{0}^{h}-\psi^{h}\right)=0$, and Lemma 3.2 applies, with the result that

$$
\left.\underset{x_{i j} \in \Omega_{R}^{\prime}}{\operatorname{Max}_{x_{i j} \in \Omega_{R}^{\prime}}\left|\partial_{2}\left(\psi_{0}^{h}-\psi^{h}\right)_{i, j}\right|}\right\} \leqslant \frac{C}{R} \underset{x_{i j} \in \Omega_{R}}{\operatorname{Max}}\left|\left(\psi_{0}^{h}-\psi^{h}\right)_{i, j}\right|
$$

Setting $\psi_{1}^{h}=\psi_{0}^{h}-\psi^{h}$, we now have to estimate $\operatorname{Max}_{x_{i,} \in \Omega_{R}}\left|\left(\psi_{1}^{h}\right)_{i, j}\right|$. The idea is to find a Neumann problem for which $\psi_{1}^{h}$ will be an approximate solution. To this end, we shall use the finite-difference formulation (2.14)-(2.16).

The function $\psi_{1}^{h}$ clearly satisfies (2.14) with a zero right-hand member. A simple calculation shows that (2.15) and (2.16) are also verified with respective right-hand members

$$
C_{i, j}=\left\{\begin{array}{l}
\frac{C_{R}(\omega)}{\varepsilon}+\frac{1}{\varepsilon}\left(\partial_{1} \psi_{0}^{h}\right)_{i+1 / 2, j}+\frac{1}{2 \varepsilon}\left(\partial_{2} \psi_{0}^{h}\right)_{i, j-1 / 2}+\frac{1}{2 \varepsilon}\left(\partial_{2} \psi_{0}^{h}\right)_{i, j+1 / 2} \\
\frac{C_{R}(\omega)}{\varepsilon}-\frac{1}{2}\left(\Delta^{\varepsilon} \psi_{0}^{h}\right)_{i, j}+\frac{1}{\varepsilon}\left(\partial_{1} \psi_{0}^{h}\right)_{i+1 / 2, j}+\frac{1}{\varepsilon}\left(\partial_{2} \psi_{0}^{h}\right)_{i, j+1 / 2}
\end{array}\right.
$$

and similar equations for the other sides and vertices of $\Omega_{R}$.

Since $C_{R}(\omega) \leqslant\|\omega\|_{L_{1}\left(\mathbf{R}^{2}\right)} / R$, and using (3.6), we observe that $\left|C_{i j}\right| \leqslant$ $C\|\omega\|_{L^{1}\left(\mathbf{R}^{2}\right)} / \varepsilon R$, where $C$ is independent of $h$ and $\omega$. We are now looking for a function $f$, piecewise constant on $\Gamma_{R}$, such that

$$
\frac{1}{\varepsilon^{2}} \int f(x) w_{\varepsilon}\left(x-x_{i j}\right) d \gamma=C_{i j} \quad \text { for all } x_{i j} \in \Gamma_{R} \text {. }
$$

For this purpose we renumber clockwise the vertices which belong to $\Gamma_{R}$,

$$
\begin{aligned}
x_{i j} & =x_{n}, \quad n \in \mathbf{N}, \quad x_{n+N}=x_{n}, \text { for all } x_{i j} \in \Gamma_{R}, \\
\varepsilon C_{i j} & =C_{n} .
\end{aligned}
$$

Let $x_{n+1 / 2}=\frac{1}{2}\left(x_{n}+x_{n+1}\right)$ and denote by $f$ the piecewise constant function defined by $f=C_{n}^{\prime}$ on $\left[x_{n-1 / 2}, x_{n+1 / 2}\right]$, where $\left(C_{n}^{\prime}\right)$ is solution of the system

$$
\frac{1}{4}\left(\frac{1}{2} C_{n-1}^{\prime}+3 C_{n}^{\prime}+\frac{1}{2} C_{n+1}^{\prime}\right)=C_{n}, \quad \text { for all } n \in \mathbf{N} \text {. }
$$


Observe that, by Hadamard's theorem, (3.9) has a unique solution. It is now obvious that $f$ satisfies (3.8) and, moreover,

$$
\|f\|_{L^{\infty}\left(\Gamma_{R}\right)}=\sup _{n}\left|C_{n}^{\prime}\right| \leqslant 2 \sup _{n}\left|C_{n}\right| \leqslant \frac{C}{R} .
$$

Then, as a consequence of (3.8), we may consider $\left(\psi_{1}^{h}\right)_{i, j}$ as the values taken at the vertices $x_{i j}$ by the $P_{1}$ finite element approximation in $\mathscr{M}_{h}$ of the solution $\psi_{1}$ of the Neumann problem

$$
\begin{cases}-\Delta \psi_{1}=0 & \text { in } \Omega_{R} \\ \frac{\partial \psi_{1}}{\partial n}=f & \text { on } \Gamma_{R}\end{cases}
$$

In order to derive $L^{\infty}$ estimates for $\psi_{1}^{h}$, we work on $\hat{\Omega}$ and define the following quantities:

$$
\hat{\psi}_{1}(\hat{x})=\frac{1}{R} \psi_{1}(R \hat{x}), \quad \hat{\psi}_{1}^{h}(\hat{x})=\frac{1}{R} \psi_{1}^{h}(R \hat{x}), \quad \hat{f}(\hat{x})=f(R \hat{x}) .
$$

Then we get

$$
\begin{cases}\Delta \hat{\psi}_{1}=0 & \text { in } \hat{\Omega}_{R} \\ \frac{\partial \hat{\psi}_{1}}{\partial n}=\hat{f} & \text { on } \hat{\Gamma}_{R}\end{cases}
$$

and, denoting still by $\hat{\psi}_{1}^{h}$ the $P_{1}$ interpolate of $\hat{\psi}_{1}^{h}$ on $\mathscr{T}_{\hat{h}}$,

for $p>2$ :

$$
\begin{aligned}
\left\|\hat{\psi}_{1}^{h}\right\|_{L^{\infty}(\hat{\Omega}) / \mathbf{R}} & \leqslant C\left\|\hat{\psi}_{1}^{h}\right\|_{W^{1, p}(\hat{\Omega})} \quad \text { (by the Sobolev inequality) } \\
& \leqslant C\left\|\hat{\psi}_{1}\right\|_{W^{1, p}(\hat{\Omega})} \\
& \leqslant C\|\hat{f}\|_{W^{-(1 / p) \cdot p(\hat{\Gamma})}} \leqslant \frac{C}{R}
\end{aligned}
$$

so that $\left\|\psi_{1}^{h}\right\|_{L^{\infty}\left(\Omega_{R}\right) / \mathbf{R}} \leqslant C$. Finally, using (3.6) and (3.7), we get

if $\left|x_{i j}\right|>2 R_{0}, x_{i j} \in \Omega_{R}^{\prime}$,

$$
\left|\left(\partial, \psi^{h}\right)_{i, j}\right| \leqslant C\left\{\frac{1}{R}+\frac{1}{\left|x_{i j}\right|}\right\} \leqslant \frac{C}{\left|x_{i j}\right|}\|\omega\|_{L^{1}\left(\mathbf{R}^{2}\right)},
$$

where $C$ is independent of $h$ and $\omega$; coming back to the definition of $\mathscr{S}^{h}$, this estimate ends the proof of the lemma.

We are now looking for more precise stability and consistency results than those obtained in Lemma 3.1. In particular, we wish to get $\varepsilon^{2}$ rather than $\varepsilon$ in the bound of (3.2). This is a superconvergence result, which is known to occur because the mesh is uniform, but the proof, for $R$ fixed, is usually based on the strong regularity of $\psi$. Therefore, the constants which appear increase with $R$.

To overcome this difficulty, we approximate $\mathscr{S}^{h}$ with a Riesz projection $\mathscr{R}^{h}$ for which we have optimal stability and consistency results.

To begin with, let us define for $\omega \in C\left(\mathbf{R}^{2}\right)$ and $\varepsilon>0$

$$
\operatorname{curl}_{\varepsilon} \omega=\frac{1}{2 \varepsilon}(\omega(x+(0, \varepsilon))-\omega(x-(0, \varepsilon)),-\omega(x+(\varepsilon, 0))+\omega(x-(\varepsilon, 0))) .
$$


Next, let $\tilde{u}$ be the solution of the following Dirichlet problem:

$$
\begin{cases}-\Delta \tilde{u}=\operatorname{curl}_{\varepsilon} \omega & \text { in } \Omega_{R}^{\prime}, \\ \tilde{u}=0 & \text { on } \Gamma_{R}^{\prime} .\end{cases}
$$

Then, we denote by $\mathscr{R}^{h}(\omega)$ the Riesz projection of $\tilde{u}$ in $\mathscr{\mathscr { M }}_{h}$, where $\mathscr{\mathscr { M }}_{h}$ is the subspace of $C\left(\bar{\Omega}_{R}^{\prime}\right)$ spanned by the family $\left\{w_{\varepsilon}\left(x-x_{i j}\right), x_{i j} \in \Omega_{R}^{\prime}\right\}$, so that $\mathscr{R}^{h}(\omega)$ satisfies

$$
\int_{\Omega_{R}^{\prime}} \nabla\left(\mathscr{R}^{h}(\omega)\right) \cdot \nabla \varphi d x=\int_{\Omega_{R}^{\prime}}\left(\operatorname{curl}_{\varepsilon} \omega\right) \varphi d x, \quad \text { all } \varphi \in \check{\mathscr{M}}_{h} .
$$

In order to make sure that $\mathscr{R}^{h}$ is well defined, we require the parameters to meet the condition $R / 4 \varepsilon \in \mathbf{N}$. The following result shows that for large $R, \mathscr{S}^{h}$ and $\mathscr{R}^{h}$ are close to each other.

Lemma 3.4. Let $\omega \in C\left(\mathbf{R}^{2}\right)$ have compact support. There exist constants $R_{0}$ and $C$ depending only on $\|\omega\|_{L^{1}\left(\mathbf{R}^{2}\right)}$ and $\rho(\omega)$ such that, if $R>R_{0}$,

$$
\begin{aligned}
& \left\|\mathscr{S}^{h}(\omega)-\mathscr{R}^{h}(\omega)\right\|_{L^{\infty}\left(\Omega_{R}^{\prime}\right)} \leqslant \frac{C}{R}, \\
& \left|\mathscr{S}^{h}(\omega)-\mathscr{R}^{h}(\omega)\right|_{1, \infty, \Omega_{R}^{\prime \prime}} \leqslant \frac{C}{R^{2}} .
\end{aligned}
$$

Proof. It is a simple matter to check that

$$
\Delta^{\varepsilon}\left(\mathscr{R}^{h}(\omega)\right)=\Delta^{\varepsilon}\left(\mathscr{S}^{h}(\omega)\right) \text { in } \Omega_{R}^{\prime} .
$$

On the other hand,

$$
\mathscr{R}^{h}(\omega)=0 \text { on } \Gamma_{R}^{\prime}
$$

and, by Lemma 3.3,

$$
\left|\mathscr{S}^{h}(\omega)\right| \leqslant \frac{C}{R} \quad \text { on } \Gamma_{R}^{\prime}, R>R_{0},
$$

where $C=C\left(\rho(\omega),\|\omega\|_{L^{1}\left(\mathbf{R}^{2}\right)}\right), R_{0}=R_{0}(\rho(\omega))$.

Lemma 3.2, assertions (ii) and (iii), now apply in $\Omega_{R}^{\prime \prime} \subset \subset \Omega_{R}^{\prime}$ to give the desired bounds.

It remains now to describe stability and consistency properties of $\mathscr{R}_{h}$.

For this purpose, we introduce the Green function

and its curl

$$
G(x)=\frac{1}{2 \pi} \log |x|, \quad x \in \mathbf{R}^{2}\{0\},
$$

Concerning stability we have

$$
K(x)=\operatorname{curl} G(x)
$$

LEMMA 3.5. Let $\omega \in C\left(\mathbf{R}^{2}\right)$ have compact support. There exist constants $C_{p}$ and $R_{0}$ depending only on $p,\|\omega\|_{L^{1}\left(\mathbf{R}^{2}\right)}$ and $\rho(\omega)$ such that, for $R>R_{0}$ and $\varepsilon<1$,

$$
\begin{gathered}
\left\|\mathscr{R}^{h}(\omega)\right\|_{L^{p}\left(\Omega_{R}^{\prime}\right)} \leqslant C_{p}\left\{\left\|G * \operatorname{curl}_{\varepsilon} \omega\right\|_{L^{p}\left(\Omega_{R}^{\prime}\right)}+\varepsilon\left|G * \operatorname{curl}_{\varepsilon} \omega\right|_{1, p, \Omega_{R}^{\prime}}\right. \\
\left.+R^{(2 / p)-1}+\varepsilon R^{(2 / p)-2}\right\}, \quad p \in[2, \infty), \\
\left|\mathscr{R}^{h}(\omega)\right|_{1, p, \Omega_{R}^{\prime}} \leqslant C_{p}\left\{R^{-1}\left\|G * \operatorname{curl}_{\varepsilon} \omega\right\|_{L^{p}\left(\Omega_{R}^{\prime}\right)}+\left|G * \operatorname{curl}_{\varepsilon} \omega\right|_{1, p, \Omega_{R}^{\prime}}\right. \\
\left.+R^{(2 / p)-2}\right\}, \quad p \in[2,+\infty] .
\end{gathered}
$$

*We denote by ||$_{l, p, \Omega}$ the usual seminorm of $W^{l, p}(\Omega), 1 \leqslant p \leqslant+\infty, l \geqslant 0$. 
Proof. Applying the results of Rannacher and Scott [9], we may write

$$
\begin{array}{ll}
\left\|\mathscr{R}^{h}(\omega)\right\|_{L^{p}\left(\Omega_{R}^{\prime}\right)} \leqslant C_{p}\left\{\|\tilde{u}\|_{L^{p}\left(\Omega_{R}^{\prime}\right)}+\varepsilon|\tilde{u}|_{1, p, \Omega_{R}^{\prime}}\right\}, & p \in[2,+\infty], \\
\left|\mathscr{R}^{h}(\omega)\right|_{1, p, \Omega_{R}^{\prime}} \leqslant C_{p}\left\{R^{-1}\|\tilde{u}\|_{L^{p}\left(\Omega_{R}^{\prime}\right)}+|\tilde{u}|_{1, p, \Omega_{R}^{\prime}}\right\}, \quad p \in[2,+\infty]
\end{array}
$$

(in fact we proceed as in the proof of Lemma 3.1 in order to use the estimates of Rannacher and Scott in $\hat{\Omega}$ ).

Next, we observe that $\tilde{u}-G * \operatorname{curl}_{\varepsilon} \omega$ satisfies, if $R$ is sufficiently large,

$$
\left\{\begin{array}{l}
\Delta\left(\tilde{u}-G * \operatorname{curl}_{\varepsilon} \omega\right)=0 \quad \text { in } \Omega_{R}^{\prime}, \\
\left|\tilde{u}-G * \operatorname{curl}_{\varepsilon} \omega\right|_{l, \infty, \Gamma_{R}^{\prime}} \leqslant \frac{C}{R^{1+l}}, \quad l \geqslant 0 .
\end{array}\right.
$$

This last bound is based on the uniform decay of $G * \operatorname{curl}_{\varepsilon} \omega$ with respet to $\varepsilon$. It could be directly proved, but will be an immediate consequence of a subsequent result (Lemma 4.4) and so will be assumed true for now.

Let us set $\psi=\tilde{u}-G * \operatorname{curl}_{\varepsilon} \omega$ and $\hat{\psi}(\hat{x})=\psi(R x / 2)$ for $\hat{x} \in \hat{\Omega}$; we obtain

$$
\|\hat{\psi}\|_{2, \infty, \hat{\Gamma}}=\|\psi\|_{L^{\infty}(\Gamma)}+R|\psi|_{1, \infty, \Gamma}+R^{2}|\psi|_{2, \infty, \Gamma}
$$

and, owing to (3.15), $\|\hat{\psi}\|_{2, \infty, \hat{\uparrow}} \leqslant C / R$.

The regularity properties of $\Delta$ ensure that

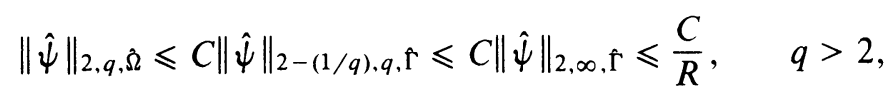

and, by the Sobolev inequalities,

$$
|\hat{\psi}|_{1, \infty, \hat{\Omega}} \leqslant\|\hat{\psi}\|_{2, q, \Omega} \leqslant \frac{C}{R} .
$$

Therefore, we obtain

$$
|\psi|_{1, \infty, \Omega_{R}^{\prime}} \leqslant \frac{C}{R^{2}} .
$$

Since, by the maximum principle, we also have

$$
\|\psi\|_{L^{\infty}\left(\Omega_{R}^{\prime}\right)} \leqslant \frac{C}{R}
$$

we finally get

$$
\left\{\begin{array}{l}
|\tilde{u}(x)| \leqslant\left|\left(G * \operatorname{curl}_{\varepsilon} \omega\right)(x)\right|+\frac{C}{R}, \\
\left|\frac{\partial}{\partial x_{i}} \tilde{u}(x)\right| \leqslant\left|\frac{\partial}{\partial x_{i}}\left(G * \operatorname{curl}_{\varepsilon} \omega\right)(x)\right|+\frac{C}{R^{2}}, \quad i=1,2 .
\end{array}\right.
$$

Consequently,

$$
\begin{aligned}
& \|\tilde{u}\|_{L^{p}\left(\Omega_{R}^{\prime}\right)} \leqslant C\left\{\left\|G * \operatorname{curl}_{\varepsilon} \omega\right\|_{L^{p}\left(\Omega_{R}^{\prime}\right)}+R^{(2 / p)-1}\right\} \\
& |\tilde{u}|_{1, p, \Omega_{R}^{\prime}} \leqslant C\left\{\left|G * \operatorname{curl}_{\varepsilon} \omega\right|_{1, p, \Omega_{R}^{\prime}}+R^{(2 / p)-2}\right\} .
\end{aligned}
$$

This, together with (3.14), yields the desired estimates. 
Combining (3.15) and optimal consistency $L^{P}$ estimates proved in [9] for $\mathscr{R}^{h}(\omega)-\tilde{u}$, we obtain

Lemмa 3.6. Assume that $\omega$ is sufficiently smooth and has compact support. Then there exist constants $C_{p}$ such that for $R$ large and $\varepsilon$ small enough,

$$
\begin{aligned}
& \left\|G * \operatorname{curl}_{\varepsilon} \omega-\mathscr{R}^{h}(\omega)\right\|_{L^{p}\left(\Omega_{R}^{\prime}\right)} \leqslant C_{p}\left\{\varepsilon^{2}+R^{(2 / p)-1}\right\}, \quad p \in[2,+\infty), \\
& \left|G * \operatorname{curl}_{\varepsilon} \omega-\mathscr{R}^{h}(\omega)\right|_{1, p, \Omega_{R}^{\prime}} \leqslant C_{p}\left\{\varepsilon+R^{(2 / p)-2}\right\}, \quad p \in[2,+\infty] .
\end{aligned}
$$

We point out that the two previous results take into account the effects of both the truncation of the plane and the finite-difference scheme. It remains now to introduce particles.

4. Convergence of the Approximation. We begin with proving Theorem 1. We use the following result.

LeMma 4.1. Let $\Omega$ be a convex domain whose boundary $\Gamma$ is Lipschitz continuous. Let $f \in L^{2}(\Omega)$ have compact support in $\Omega$ and let $a \in \mathbf{R}^{2}$. Then the Neumann problem

$$
\begin{cases}-\Delta \psi=(a \cdot \nabla) f & \text { in } \Omega \\ \frac{\partial \psi}{\partial n}=0 & \text { on } \Gamma\end{cases}
$$

has a unique solution $\psi$ in $H^{1}(\Omega) / \mathbf{R}$ and $\|\psi\|_{H^{1}(\Omega) / \mathbf{R}} \leqslant C\|f\|_{L^{2}(\Omega)}$.

Proof. We consider the following variational problem:

$$
\left\{\begin{array}{l}
\text { Find } \psi \in H^{1}(\Omega) / \mathbf{R} \text { such that } \\
\int_{\Omega} \nabla \psi \cdot \nabla \varphi d x=\int_{\Omega} f \cdot(a \cdot \nabla) \varphi d x, \quad \text { all } \varphi \in H^{1}(\Omega) / \mathbf{R} .
\end{array}\right.
$$

The bilinear form in the left-hand member is elliptic, which proves the existence and the uniqueness of the solution $\psi$. It is now readily seen that, since $f$ has its support in $\Omega$, our variational problem can be regarded as a formulation of the Neumann problem (4.1).

We now present the

Proof of Theorem 1. It suffices to check that the system of ordinary differential equations

$$
\left\{\begin{array}{l}
\frac{d X_{k}^{h}}{d s}=\vec{u}^{h}\left(X_{k}^{h}(s), s\right), \\
X_{k}^{h}(0)=x_{k}
\end{array}\right.
$$

admits a unique solution in $[0, T]$. Let

$$
B=\left\{\left(X_{k}\right)_{|k| \leqslant N} \in\left(l^{\infty}(0, T)\right)^{N^{2}} \text {, all } s \in[0, T]: X_{k}(s) \in \Omega_{R-1}\right\} .
$$

We prove that (4.2) is Lipschitzian in $B$. For the sake of simplicity we drop the subscripts $h, \varepsilon$ and $R$ (the constants occurring in the sequel will depend on these parameters, of course). Making explicit how $\vec{u}^{h}$ depends on $X^{h}$, we can rewrite (4.2) as

$$
\left\{\begin{array}{l}
\frac{d X_{k}}{d s}=\mathscr{S}\left(\sum_{k} \alpha_{k} \chi\left(x-X_{k}(s)\right)\right)\left(X_{k}(s), s\right), \\
X_{k}(0)=x_{k}
\end{array}\right.
$$


We have thus to estimate

$$
\mathscr{S}\left(\sum_{k} \alpha_{k} \chi\left(x-X_{k}(s)\right)\right)\left(X_{k}(s), s\right)-\mathscr{S}\left(\sum_{k} \alpha_{k} \chi\left(x-X_{k}^{\prime}(s)\right)\right)\left(X_{k}^{\prime}(s), s\right) .
$$

Let us set

$$
\omega(x, s)=\sum_{k} \alpha_{k} \chi\left(x-X_{k}(s)\right), \quad \omega^{\prime}(x, s)=\sum_{k} \alpha_{k} \chi\left(x-X_{k}^{\prime}(s)\right) .
$$

We get

$$
\begin{aligned}
& \left|\mathscr{S}(\omega)\left(X_{k}(s), s\right)-\mathscr{S}\left(\omega^{\prime}\right)\left(X_{k}^{\prime}(s), s\right)\right| \\
& \quad \leqslant\left|\left(\mathscr{S}(\omega)-\mathscr{S}\left(\omega^{\prime}\right)\right)\left(X_{k}(s), s\right)\right|+\left|\mathscr{S}\left(\omega^{\prime}\right)\left(X_{k}(s), s\right)-\mathscr{S}\left(\omega^{\prime}\right)\left(X_{k}^{\prime}(s), s\right)\right| \\
& \quad \leqslant\left\|\mathscr{S}\left(\omega-\omega^{\prime}\right)\right\|_{L^{\infty}(\Omega)}+\left\|\mathscr{S}\left(\omega^{\prime}\right)\right\|_{W^{1, \infty}(\Omega)} \operatorname{Max}_{k}\left|X_{k}(s)-X_{k}^{\prime}(s)\right| .
\end{aligned}
$$

Since $\mathscr{S}\left(\omega-\omega^{\prime}\right) \in \mathscr{M}$, which is a finite-dimensional subspace of $W^{1, \infty}(\Omega)$, we can write

$$
\left\|\mathscr{S}\left(\omega-\omega^{\prime}\right)\right\|_{L^{\infty}(\Omega)} \leqslant C\left\|\mathscr{S}\left(\omega-\omega^{\prime}\right)\right\|_{L^{2}(\Omega)}
$$

and, by a classical stability result,

$$
\left\|\mathscr{S}\left(\omega-\omega^{\prime}\right)\right\|_{L^{2}(\Omega)} \leqslant C\|\psi\|_{H^{1}(\Omega) / \mathbf{R}},
$$

where $\psi$ denotes the solution of the Neumann problem

$$
\left\{\begin{array}{l}
-\Delta \psi=\omega-\omega^{\prime} \\
\frac{\partial \psi}{\partial n}=C\left(\omega-\omega^{\prime}\right)
\end{array}\right.
$$

Since $\left(X_{k}\right) \in B,\left(X_{k}^{\prime}\right) \in B$, we have

$$
C(\omega)=C\left(\omega^{\prime}\right)=\sum_{k} \alpha_{k}
$$

On the other hand, we can write

$$
\begin{aligned}
& \left(\omega-\omega^{\prime}\right)(x)=\sum_{k} \alpha_{k}\left(\chi\left(x-X_{k}(s)\right)-\chi\left(x-X_{k}^{\prime}(s)\right)\right) \\
& =\sum_{k} \alpha_{k}\left[X_{k}(s)-X_{k}^{\prime}(s)\right] \cdot \nabla \int_{0}^{1} \chi\left[x-X_{k}(s)+t\left(X_{k}^{\prime}(s)-X_{k}(s)\right)\right] d t,
\end{aligned}
$$

and Lemma 4.1 applies, giving the bound

$$
\begin{aligned}
\|\psi\|_{H^{1}(\Omega) / \mathbf{R}} & \leqslant C\left(\sum_{k}\left|\boldsymbol{\alpha}_{k}\right|\right) \underset{k}{\operatorname{Max}}\left|X_{k}(s)-X_{k}^{\prime}(s)\right|\|\chi\|_{L^{\infty}\left(\mathbf{R}^{2}\right)}|\Omega|^{1 / 2} \\
& \leqslant C \operatorname{Max}_{k}\left|X_{k}(s)-X_{k}^{\prime}(s)\right| .
\end{aligned}
$$

Hence, by (4.3) and (4.4), we get

$$
\left\|\mathscr{S}\left(\omega-\omega^{\prime}\right)\right\|_{L^{\infty}(\Omega)} \leqslant C \operatorname{Max}_{k}\left|X_{k}(s)-X_{k}^{\prime}(s)\right|
$$

and, finally,

$$
\left|\mathscr{S}(\omega)\left(X_{k}(s), s\right)-\mathscr{S}\left(\omega^{\prime}\right)\left(X_{k}^{\prime}(s), s\right)\right| \leqslant C \operatorname{Max}_{k}\left|X_{k}(s)-X_{k}^{\prime}(s)\right| .
$$


Next, since (4.2) is Lipschitzian we know that (4.2) has a unique maximal solution in $\left[0, T^{*}\right] \subset[0, T]$. Suppose now that $T^{*} \neq T$; then there exists some index $k$ such that $X_{k}\left(T^{*}\right) \in \partial B$. But if $R$ is chosen sufficiently large, this cannot occur because of the definition of $\mathscr{S}^{h}$, which ensures that $\vec{u}^{h}=0$ if $x \notin \bar{\Omega}_{3 R / 4+\varepsilon}$.

If $R>\rho\left(\omega_{0}\right)+1$ and if $\frac{3}{4} R+\varepsilon<R-1$, we have

$$
\left\{\begin{array}{l}
X_{k}(0) \in \Omega_{R-1}, \\
\vec{u}^{h}(x)=0 \text { if } x \notin \Omega_{R-1}
\end{array}\right.
$$

and therefore (provided $\varepsilon<\frac{1}{2}$ )

$$
X_{k}\left(T^{*}\right) \in \Omega_{R-1}
$$

completing the proof.

We now come to the convergence itself. The basic point is to write the error $\vec{u}-\vec{u}^{h}$ in the following way:

$$
\begin{aligned}
\vec{u}-\vec{u}^{h}= & {\left[K * \omega-\mathscr{R}^{h}(\omega)\right]+\left[\mathscr{R}^{h}\left(\omega-\tilde{\omega}^{h}\right)\right] } \\
& +\left[\mathscr{R}^{h}\left(\tilde{\omega}^{h}\right)-\mathscr{S}^{h}\left(\tilde{\omega}^{h}\right)\right] \text { in } \Omega_{R}^{\prime},
\end{aligned}
$$

where $\tilde{\omega}^{h}$ was defined in (2.10) and $\mathscr{R}^{h}$ in Section 3.

In order to estimate the third bracket, the following result will allow us to apply Lemma 3.4.

Lemma 4.2. Assume that $\operatorname{Max}_{j}\left|X_{j}(t)-X_{j}^{h}(t)\right|<1$ for all $t$ in $[0, T]$. Then there exists a constant $C$ such that

$$
\begin{aligned}
& \left\|\tilde{\omega}^{h}(\cdot, t)\right\|_{L^{1}\left(\mathbf{R}^{2}\right)} \leqslant C, \quad t \in[0, T] . \\
& \rho\left(\tilde{\omega}^{h}(\cdot, t)\right) \leqslant C,
\end{aligned}
$$

Proof. Since $\left\|\chi_{\varepsilon}\right\|_{L^{1}\left(\mathbf{R}^{2}\right)}=1$ we clearly have

$$
\left\|\tilde{\omega}^{h}(\cdot, t)\right\|_{L^{1}\left(\mathbf{R}^{2}\right)} \leqslant \sum_{j}\left|\alpha_{j}\right|
$$

On the other hand, it is readily seen that

$$
\rho\left(\tilde{\omega}^{h}(\cdot, t)\right) \leqslant \operatorname{Max}_{j}\left|X_{j}(t)-X_{j}^{h}(t)\right|+\varepsilon+\rho(\omega(\cdot, t)) \leqslant C .
$$

Lemma 3.4, therefore, can be applied to give

LEMma 4.3. Under the assumption that $\operatorname{Max}_{j}\left|X_{j}(t)-X_{j}^{h}(t)\right|<1$ for $t$ in $[0, T]$, we have for $R>R_{0}(T)$

$$
\begin{gathered}
\left\|\mathscr{R}^{h}\left(\tilde{\omega}^{h}(\cdot, t)\right)-\mathscr{S}^{h}\left(\tilde{\omega}^{h}(\cdot, t)\right)\right\|_{L^{p}\left(\Omega_{R}^{\prime \prime}\right)} \leqslant C_{p} R^{(2 / p)-1}, \\
\left|\mathscr{R}^{h}(\tilde{\omega}(\cdot, t))-\mathscr{S}^{h}\left(\tilde{\omega}^{h}(\cdot, t)\right)\right|_{1, p, \Omega_{R}^{\prime \prime}} \leqslant C_{p} R^{(2 / p)-2}, \quad p \in[2,+\infty] .
\end{gathered}
$$

Next, we rewrite the first bracket in (4.5):

$$
K * \omega-\mathscr{R}^{h}(\omega)=\left(K * \omega-G * \operatorname{curl}_{\varepsilon} \omega\right)+\left(G * \operatorname{curl}_{\varepsilon} \omega-\mathscr{R}^{h}(\omega)\right) .
$$

On the one hand, Lemma 3.6 gives an estimate for $\left(G * \operatorname{curl}_{\varepsilon} \omega-\mathscr{R}^{h}(\omega)\right)$. On the other hand, we can derive an estimate for $\left(K * \omega-G * \operatorname{curl}_{\varepsilon} \omega\right)$ from the following result, whose proof can easily be obtained from the arguments given in [10], for instance. 
LEMMA 4.4. Let $\mu$ be a measure in $\mathbf{R}^{2}$ such that

$$
\langle 1, \mu\rangle=1, \quad\left\langle x_{1}, \mu\right\rangle=\left\langle x_{2}, \mu\right\rangle=0,
$$

and, for $\varepsilon>0$, let the measure $\mu_{\varepsilon}$ be defined by $\left\langle\mu_{\varepsilon}, \varphi\right\rangle=\langle\mu, \varphi(\cdot \varepsilon)\rangle$.

Then for $f \in W^{1,1}\left(\mathbf{R}^{2}\right) \cap W^{1, \infty}\left(\mathbf{R}^{2}\right)$ with compact support, we have

$$
\left\|K * f-K * \mu_{\varepsilon} * f\right\|_{L^{p}\left(\mathbf{R}^{2}\right)} \leqslant C_{p} \varepsilon^{2}
$$

We first rewrite $G * \operatorname{curl}_{\varepsilon} \omega$ as $\operatorname{curl}_{\varepsilon} G * \omega$. Then a straightforward calculation gives

$$
\operatorname{curl}_{\varepsilon} G=K *\left(\delta \oplus \mathbf{1}_{\varepsilon}, \mathbf{1}_{\varepsilon} \oplus \delta\right) \text {, }
$$

where $\oplus$ denotes the tensor product in $\mathbf{R}^{2}$ and

$$
\begin{gathered}
\mathbf{1}_{\varepsilon}(x)=\frac{1}{2 \varepsilon} \mathbf{1}\left(\frac{x}{\varepsilon}\right), \\
\mathbf{1}=\text { characteristic function of }[-1,+1] .
\end{gathered}
$$

Letting now $\mu=\frac{1}{2}(\delta \oplus 1,1 \oplus \delta)$, we are in the situation of Lemma 4.4. We have therefore proved the following result.

LEMMA 4.5. Assume that $\omega_{0} \in W^{1,1}\left(\mathbf{R}^{2}\right) \cap W^{1, \infty}\left(\mathbf{R}^{2}\right)$ with compact support. There exist constants $C_{p}=C_{p}(T)$ such that for $\varepsilon$ small enough, $R$ large enough and $t \in[0, T]$,

$$
\begin{array}{cc}
\left\|(K * \omega)(\cdot, t)-\mathscr{R}^{h}(\omega)(\cdot, t)\right\|_{L^{p}\left(\Omega_{R}^{\prime \prime}\right)} \leqslant C_{p}\left\{\varepsilon^{2}+R^{(2 / p)-1}\right\}, & p \in[2,+\infty), \\
\left|(K * \omega)(\cdot, t)-\mathscr{R}^{h}(\omega)(\cdot, t)\right|_{1, p, \Omega_{R}^{\prime \prime}} \leqslant C_{p}\left\{\varepsilon+R^{(2 / p)-2}\right\}, & p \in[2,+\infty] .
\end{array}
$$

The observation made in (3.15) concerning the decay of $G * \operatorname{curl}_{\varepsilon} \omega$ is also clear now. Writing

$$
\operatorname{curl}_{\varepsilon} G * \omega=K *\left[\omega *\left(\delta \oplus \mathbf{1}_{\varepsilon}, \mathbf{1}_{\varepsilon} \oplus \delta\right)\right]=K * \omega^{\varepsilon}
$$

and using the decay of $K$, we get

$$
\left|\partial^{\prime}\left(\operatorname{curl}_{\varepsilon} G * \omega\right)(x)\right| \leqslant C\left(\rho\left(\omega^{\varepsilon}\right),\left\|\omega^{\varepsilon}\right\|_{L^{1}\left(\mathbf{R}^{2}\right)}\right) \frac{1}{|x|^{l+1}} \quad \text { if }|x|>\rho\left(\omega^{\varepsilon}\right), l \geqslant 0 .
$$

But we clearly have

$$
\rho\left(\omega^{\varepsilon}\right) \leqslant \rho(\omega)+\varepsilon \text { and }\left\|\omega^{\varepsilon}\right\|_{L^{1}\left(\mathbf{R}^{2}\right)}=\|\omega\|_{L^{1}\left(\mathbf{R}^{2}\right)},
$$

so that (3.15) is satisfied.

We also point out that writing (4.6) is just one way to express the regularization effect of the finite-difference scheme.

The term $\mathscr{R}^{h}\left(\omega-\tilde{\omega}^{h}\right)$ is more difficult to estimate. Before applying Lemma 3.5, we need a bound for $G * \operatorname{curl}_{\varepsilon}\left(\omega-\tilde{\omega}^{h}\right)$. Using $\tilde{\omega}^{h}=\omega^{h} * \chi_{\varepsilon}$, we can write

$$
\operatorname{curl}_{\varepsilon} G *\left(\omega-\tilde{\omega}^{h}\right)=\operatorname{curl}_{\varepsilon} G * \omega-\left(\operatorname{curl}_{\varepsilon} G * \chi_{\varepsilon}\right) * \omega^{h} \text {. }
$$

We now set

$$
K_{\varepsilon}=\operatorname{curl}_{\varepsilon} G * \chi_{\varepsilon}
$$

Then,

$$
\operatorname{curl}_{\varepsilon} G *\left(\omega-\tilde{\omega}^{h}\right)=K_{\varepsilon} *\left(\omega-\omega^{h}\right)+\left(\operatorname{curl}_{\varepsilon} G-K_{\varepsilon}\right) * \omega .
$$

Applying Lemma 4.4 successively with

$$
f=\left(\delta \oplus \mathbf{1}_{\varepsilon}, \mathbf{1}_{\varepsilon} \oplus \delta\right) * \omega \quad \text { and } \quad f=\left(\delta \oplus \mathbf{1}_{\varepsilon}, \mathbf{1}_{\varepsilon} \oplus \delta\right) * \frac{\partial \omega}{\partial x_{l}}, \quad l=1,2
$$


we get, if $\omega$ is sufficiently smooth,

$$
\left\|\left(\operatorname{curl}_{\varepsilon} G-K_{\varepsilon}\right) * \omega\right\|_{W^{1, p}\left(\mathbf{R}^{2}\right)} \leqslant C_{p} \varepsilon^{2} .
$$

Now estimating $K_{\varepsilon} *\left(\omega-\omega^{h}\right)$ amounts, on the one hand, to investigating the regularity properties of the kernel $K_{\varepsilon}$, and on the other hand, to making use of techniques involved in particle methods with nonsmooth cutoff.

We first establish

LEMMA 4.6. The following assertions hold for $\varepsilon>0$ :

(i) $K_{\varepsilon} \in W^{2, p}\left(\mathbf{R}^{2}\right) \cap \mathscr{C}^{\infty}\left(\mathbf{R}^{2}-\{x,|x| \leqslant 2 \varepsilon\}\right), p \in(2,+\infty)$,

(ii) $\left|K_{\varepsilon}\right|_{l, \infty, \mathbf{R}^{2}} \leqslant C / \varepsilon^{l+1}, l=0,1$,

(iii) $\left|K_{\varepsilon}\right|_{l, p, \mathbf{R}^{2}} \leqslant C_{p} / \varepsilon^{l+1-(2 / p)}, l=0,1, p \in(2,+\infty)$,

(iv) $\left|\partial^{\prime} K_{\varepsilon}(x)\right| \leqslant C /|x|^{l+1}, l \geqslant 0,|x| \geqslant 2 \varepsilon$.

Proof. Denote by $K^{1}, K^{2}, K_{\varepsilon}^{1}, K_{\varepsilon}^{2}$ the components of $K$ and $K_{\varepsilon}$. We shall prove our assertions for $K_{\varepsilon}^{1}$ :

$$
K_{\varepsilon}^{1}=\frac{\partial G}{\partial x_{2}} *\left(\delta \oplus 1_{\varepsilon}\right) * \chi_{\varepsilon}=K^{1} *\left(\delta \oplus 1_{\varepsilon}\right) * \chi_{\varepsilon} .
$$

Using that fact that $\partial K^{1} / \partial x_{1}=-\partial K^{2} / \partial x_{2}$, we can write the derivatives of $K_{\varepsilon}$ of order one and two in the following way:

$$
\begin{gathered}
\frac{\partial K_{\varepsilon}^{1}}{\partial x_{1}}=-\frac{\partial K^{2}}{\partial x_{2}} *\left(\delta \oplus \mathbf{1}_{\varepsilon}\right) * \chi_{\varepsilon}=-K^{2} *\left(\delta \oplus \frac{\partial \mathbf{1}_{\varepsilon}}{\partial x_{2}}\right) * \chi_{\varepsilon}, \\
\frac{\partial K_{\varepsilon}^{1}}{\partial x_{2}}=\frac{\partial K^{1}}{\partial x_{2}} *\left(\delta \oplus \mathbf{1}_{\varepsilon}\right) * \chi_{\varepsilon} \\
\frac{\partial^{2} K_{\varepsilon}^{1}}{\partial x_{1}^{2}}=-\frac{\partial K^{2}}{\partial x_{1}} *\left(\delta \oplus \frac{\partial \mathbf{1}_{\varepsilon}}{\partial x_{2}}\right) * \chi_{\varepsilon} \\
\frac{\partial^{2} K_{\varepsilon}^{1}}{\partial x_{1} \partial x_{2}}=\frac{\partial K^{1}}{\partial x_{1}} *\left(\delta \oplus \frac{\partial \mathbf{1}_{\varepsilon}}{\partial x_{2}}\right) * \chi_{\varepsilon} \\
\frac{\partial^{2} K_{\varepsilon}^{1}}{\partial x_{2}^{2}}=\frac{\partial K^{1}}{\partial x_{2}} *\left(\delta \oplus \frac{\partial \mathbf{1}_{\varepsilon}}{\partial x_{2}}\right) * \chi_{\varepsilon}
\end{gathered}
$$

Next we have

$$
\left.\begin{array}{l}
\left(\delta \oplus \mathbf{1}_{\varepsilon}\right) * \chi_{\varepsilon} \\
\left(\delta \oplus \frac{\partial}{\partial x_{2}} \mathbf{1}_{\varepsilon}\right) * \chi_{\varepsilon}
\end{array}\right\} \in L^{\infty}\left(\mathbf{R}^{2}\right), \quad \text { with support in }\left[-\frac{3 \varepsilon}{2}, \frac{3 \varepsilon}{2}\right]^{2}
$$

and

$$
\begin{gathered}
\left\|\left(\delta \oplus \mathbf{1}_{\varepsilon}\right) * \chi_{\varepsilon}\right\|_{L^{p}\left(\mathbf{R}^{2}\right)} \leqslant \frac{C}{\varepsilon^{2-(2 / p)}}, \\
\left\|\left(\delta \oplus \frac{\partial}{\partial x_{2}} \mathbf{1}_{\varepsilon}\right) * \chi_{\varepsilon}\right\|_{L^{p}\left(\mathbf{R}^{2}\right)} \leqslant \frac{C}{\varepsilon^{3-(2 / p)}}, \quad 1 \leqslant p \leqslant+\infty .
\end{gathered}
$$

Assertions (i) and (ii) now result from classical calculations based on the regularity of $K$ away from 0 and on its decay. On the other hand, owing to (4.10), Calderón's theorem applies to prove our assertion (iii). 
We are now in a position to estimate the first term in the right-hand member of (4.8), which represents the particle part of the error. As is familiar in particle methods, we introduce the following notations. Denoting by $\left(X_{k}\right)_{k}$ the exact characteristic curves, i.e., the solutions of

$$
\left\{\begin{array}{l}
\frac{d X_{k}}{d s}=\vec{u}\left(X_{k}, s\right) \\
X_{k}(0)=x_{k}
\end{array}\right.
$$

and by $\left(X_{k}^{h}\right)_{k}$ the approximate characteristic curves defined in (2.2), we set

$$
\begin{gathered}
e_{k}(t)=\left|X_{k}(t)-X_{k}^{h}(t)\right|, \\
\|e(t)\|_{\infty}=\operatorname{Max}_{k}\left|X_{k}(t)-X_{k}^{h}(t)\right|, \\
\|e(t)\|_{p}=\left\{\sum_{k}\left|B_{k} \| X_{k}(t)-X_{k}^{h}(t)\right|^{p}\right\}^{1 / p}, \quad 1 \leqslant p<\infty .
\end{gathered}
$$

We then have the following result, based on the regularity properties established in Lemma 4.6, and whose proof can be found in [3]:

Lemma 4.7. Let $T>0$. There exist constants $C_{p}, \varepsilon_{0}, \Delta x_{0}$ such that for $t \in[0, T]$, $\varepsilon<\varepsilon_{0}, \Delta x<\Delta x_{0}, \Delta x / \varepsilon \leqslant C$ and $p \in(2,+\infty)$ :

$$
\left\|K_{\varepsilon} *\left(\omega-\omega^{h}\right)(\cdot, t)\right\|_{L^{p}\left(\mathbf{R}^{2}\right)} \leqslant C_{p}\left\{\frac{\Delta x^{2}}{\varepsilon}+\frac{\Delta x^{3}}{\varepsilon^{2}}+\left(1+\frac{\|e(t)\|_{\infty}}{\varepsilon}\right)\|e(t)\|_{p}\right\},
$$

$$
\begin{gathered}
\left|K_{\varepsilon} *\left(\omega-\omega^{h}\right)(\cdot, t)\right|_{1, p, \mathbf{R}^{2}} \leqslant \frac{C_{p}}{\varepsilon}\left\{\Delta x+\frac{\Delta x^{2}}{\varepsilon}+\left(1+\frac{\|e(t)\|_{\infty}}{\varepsilon}\right)\|e(t)\|_{p}\right\}, \\
\left\|K_{\varepsilon} * \omega^{h}(\cdot, t)\right\|_{1, \infty, \mathbf{R}^{2}} \leqslant C\left\{1+\frac{\|e(t)\|_{\infty}}{\varepsilon}\right\}^{2}
\end{gathered}
$$

An immediate consequence of this result is

LEMMA 4.8. Under the assumptions of Lemma 4.7, and for $R$ large enough, we have

(i)

$$
\begin{aligned}
& \left\|\mathscr{R}^{h}\left(\omega-\tilde{\omega}^{h}\right)(\cdot, t)\right\|_{L^{p}\left(\Omega_{R}^{\prime}\right)} \\
& \leqslant C_{p}\left\{\varepsilon^{2}+\frac{\Delta x^{2}}{\varepsilon}+\frac{\Delta x^{3}}{\varepsilon^{2}}+R^{(2 / p)-1}+\left(1+\frac{\left.\left.\|e(t)\|_{\infty}\right)\|e(t)\|_{p}\right\},}{\varepsilon}\right)\right. \\
& \left.\left|\mathscr{R}^{h}\left(\omega-\tilde{\omega}^{h}\right)(\cdot, t)\right|_{1, p, \Omega_{R}^{\prime}}\right) \\
& \leqslant \frac{C_{p}}{\varepsilon}\left\{\varepsilon^{2}+\Delta x+\frac{\Delta x^{2}}{\varepsilon}+\left(1+\frac{\|e(t)\|_{\infty}}{\varepsilon}\right)\|e(t)\|_{p}\right\}, \\
& \left|\mathscr{R}^{h}\left(\tilde{\omega}^{h}\right)(\cdot, t)\right|_{1, \infty, \Omega_{R}^{\prime}} \leqslant C\left\{1+\frac{\|e(t)\|_{\infty}}{\varepsilon}\right\} .
\end{aligned}
$$

Finally, we have the following result which completes the study of the stability of the method. 
LEMMA 4.9. Under the assumptions of Lemma 4.7 we have for $t \in[0, T]$

$$
\left|u^{h}(\cdot, t)\right|_{1, \infty, \Omega_{R}^{\prime \prime}} \leqslant C\left\{1+\frac{\|e(t)\|_{\infty}}{\varepsilon}\right\}^{2} .
$$

Proof. We write

$$
\left|u^{h}(\cdot, t)\right|_{1, \infty, \Omega_{R}^{\prime \prime}} \leqslant\left|\mathscr{R}^{h}\left(\tilde{\omega}^{h}\right)\right|_{1, \infty, \Omega_{R}^{\prime \prime}}+\left|\mathscr{R}^{h}\left(\tilde{\omega}^{h}\right)-\mathscr{S}^{h}\left(\tilde{\omega}^{h}\right)\right|_{1, \infty, \Omega_{R}^{\prime \prime}}
$$

Lemma 4.8, assertion (iii), applies to bound the first term and Lemma 3.4 to bound the second.

Having obtained estimates for each of the terms in the right-hand member of (4.5), we are now in a position to give the

Proof of Theorem 2. Let $y_{p}(t)=\int_{0}^{t}\|e(\theta)\|_{p} d \theta$. From the definition of characteristics, we get

$$
\begin{aligned}
\frac{d}{d t} y_{p}(t) \leqslant & \int_{0}^{t}\left\{\sum_{k}\left|B_{k} \|\left(\vec{u}-\vec{u}^{h}\right)\left(X_{k}(\theta), \theta\right)\right|^{p}\right\}^{1 / p} d \theta \\
& +\int_{0}^{t}\left\{\sum_{k}\left|B_{k} \| \vec{u}^{h}\left(X_{k}(\theta), \theta\right)-\vec{u}^{h}\left(X_{k}^{h}(\theta), \theta\right)\right|^{p}\right\}^{1 / p} d \theta .
\end{aligned}
$$

Denote by $E_{1}, E_{2}, E_{3}$ the different brackets occurring in (4.5),

$$
E_{1}=K * \omega-\mathscr{R}^{h}(\omega), \quad E_{2}=\mathscr{R}^{h}\left(\omega-\tilde{\omega}^{h}\right), \quad E_{3}=\mathscr{R}^{h}\left(\tilde{\omega}^{h}\right)-\mathscr{S}^{h}\left(\tilde{\omega}^{h}\right) .
$$

First we have

$$
\left\|E_{3}\right\|_{L^{\infty}\left(\Omega_{R}^{\prime \prime}\right)} \leqslant \frac{C}{R^{2}}
$$

Hence,

$$
\left\{\sum_{k}\left|B_{k}\right|\left|E_{3}\left(X_{k}(t), t\right)\right|^{p}\right\}^{1 / p} \leqslant \frac{C}{R^{2}} .
$$

Next, using classical tools from interpolation theory, we get

$$
\begin{aligned}
& \left\{\sum_{\left(k, X_{k}(t) \in \Omega_{R}^{\prime \prime}\right)}\left|B_{k} \| E_{2}\left(X_{k}(t), t\right)\right|^{p}\right\}^{1 / p} \\
& \leqslant C\left\{\left\|E_{2}(\cdot, t)\right\|_{L^{p}\left(\Omega_{R}^{\prime \prime}\right)}+\Delta x\left|E_{2}(\cdot, t)\right|_{1, p, \Omega_{R}^{\prime \prime}}\right\}, \\
& \left\{\sum_{\left(k, X_{k}(t) \in \Omega_{R}^{\prime \prime}\right)}\left|B_{k} \| E_{1}\left(X_{k}(t), t\right)\right|^{p}\right\}^{1 / p} \\
& \quad \leqslant C\left\{\left\|E_{1}(\cdot, t)\right\|_{L^{p}\left(\Omega_{R}^{\prime \prime}\right)}+\Delta x\left|E_{1}(\cdot, t)\right|_{1, p, \Omega_{R}^{\prime \prime}}\right\} .
\end{aligned}
$$

Lemmas $4.5,4.8$ now apply to bound $\left\{\sum_{\left(k, X_{k}(t)\right)}\left|B_{k} \|\left(E_{1}+E_{2}\right)\left(X_{k}(t), t\right)\right|^{p}\right\}^{1 / p}$. Together with (4.12), this gives

$$
\begin{aligned}
& \left\{\sum_{\left(k, X_{k}(t) \in \Omega_{R}^{\prime \prime}\right)}\left|B_{k} \|\left(\vec{u}-\vec{u}^{h}\right)\left(X_{k}(t), t\right)\right|^{p}\right\}^{1 / p} \\
& \quad \leqslant C\left\{\varepsilon^{2}+\frac{\Delta x^{2}}{\varepsilon}+R^{(2 / p)-1}+\left(1+\frac{\|e(t)\|_{\infty}}{\varepsilon}\right)\|e(t)\|_{p}\right\} .
\end{aligned}
$$


Now if $X_{k}(t) \notin \Omega_{R}^{\prime \prime}$ we get

$$
\left|\left(\vec{u}-\vec{u}^{h}\right)\left(X_{k}(t), t\right)\right| \leqslant \frac{C}{R} .
$$

Using Lemma 4.9 and the decay of $\vec{u}^{h}$ out of $\Omega_{R}^{\prime \prime}$, we have

$$
\begin{gathered}
\left\{\sum_{k}\left|B_{k} \| \vec{u}^{h}\left(X_{k}(t), t\right)-\vec{u}^{h}\left(X_{k}^{h}(t), t\right)\right|^{p}\right\}^{1 / p} \\
\leqslant C\left\{\left(1+\frac{\|e(t)\|_{\infty}}{\varepsilon}\right)^{2}\|e(t)\|_{p}+\frac{1}{R}\right\} .
\end{gathered}
$$

The bounds (4.13)-(4.15) enable us to estimate the right-hand member of (4.11). In order to apply Gronwall's inequality, we define

$$
T_{h}^{*}=\sup \left\{t \in[0, T],\|e(t)\|_{\infty} \leqslant \varepsilon\right\} .
$$

We have

$$
y_{p}^{\prime}(t) \leqslant C_{p}\left(\varepsilon^{2}+\frac{\Delta x^{2}}{\varepsilon}+R^{(2 / p)-1}+y_{p}(t)\right), \quad t \in\left[0, T_{h}^{*}\right],
$$

where $C_{p}$ depends only on $T, p$ and the exact solution $\vec{u}$.

We therefore obtain

$$
y_{p}(t) \leqslant C\left\{\varepsilon^{2}+\frac{\Delta x^{2}}{\varepsilon}+R^{(2 / p)-1}\right\}, \quad t \in\left[0, T_{h}^{*}\right] .
$$

We now prove that $T_{h}^{*}=T$.

Clearly, we have

$$
\|e(t)\|_{\infty} \leqslant\|e(t)\|_{p} \Delta x^{-2 / p}
$$

Hence,

$$
\begin{aligned}
\left\|e\left(T_{h}^{*}\right)\right\|_{\infty} & \leqslant C_{p}\left\{\varepsilon^{2} \Delta x^{-2 / p}+\frac{\Delta x^{2-(2 / p)}}{\varepsilon}+R^{(2 / p)-1} \Delta x^{-2 / p}\right\} \\
& \leqslant C_{p}\left\{\varepsilon^{2-(2 s / p)}+\varepsilon^{2 s-(2 s / p)-1}+\varepsilon^{s-(2 s / p)}\right\},
\end{aligned}
$$

provided $\Delta x, \varepsilon$ and $R$ meet the conditions given in the theorem. Choosing $p$ such that

$$
s-\frac{2 s}{p} \geqslant \alpha>1, \quad 2-\frac{2 s}{p} \geqslant \alpha>1
$$

we get

$$
\left\|e\left(T_{h}^{*}\right)\right\| \leqslant C_{p} \varepsilon^{\alpha}<\frac{\varepsilon}{2}
$$

if $\varepsilon$ is small enough. Since $X_{k}$ and $X_{k}^{h}$ are continuous functions of time, this is impossible unless $T_{h}{ }^{*}=T$.

Finally, (4.16) in turn leads to the following bound (via estimates of $E_{1}, E_{2}, E_{3}$ in $\Omega_{R}^{\prime \prime}$ and the decay of $\vec{u}$ and $\vec{u}^{h}$ out of $\left.\Omega_{R}^{\prime \prime}\right)$ :

$$
\left\|\vec{u}-\vec{u}^{h}(\cdot, t)\right\|_{L^{p}\left(\mathbf{R}^{2}\right)} \leqslant C\left\{\varepsilon^{2}+\frac{\Delta x^{2}}{\varepsilon}+R^{(2 / p)-1}\right\}, \quad p \in(2,+\infty),
$$

and the proof is completed. 
5. Conclusion. We have proved the convergence of a Vortex In Cell method in which, in order to compute velocity in one grid point, we need the vorticity carried by particles lying in the 16 closest cells.

It is natural to wonder whether or not methods involving fewer particles converge. In fact, from the proof given in this paper, it is clear that the convergence of the conventional CIC method (in which (2.11) is replaced by $u^{h}=\mathscr{S}^{h}\left(\omega^{h}\right)$ ) is related to the convergence of Vortex methods with cutoff functions $\zeta$ such that

$$
\left\{\begin{array}{l}
\zeta \in L^{1}\left(\mathbf{R}^{2}\right) \cap L^{\infty}\left(\mathbf{R}^{2}\right) \\
\frac{\partial \zeta}{\partial x_{1}}=\mathbf{1} \oplus \delta ; \quad \frac{\partial \zeta}{\partial x_{2}}=\delta \oplus \mathbf{1} .
\end{array}\right.
$$

The author has proved, in unpublished work based on a slight modification of the results given in [3] (in particular of the stability analysis), that (5.1) actually leads to a convergent method, a result whose consequence is the convergence of the CIC method.

Finally, we observe that the role of the condition $R>R_{0}$ appearing in the proof of convergence is, in particular, to guarantee that particles cannot leave the computational domain $\Omega_{R}$. In fact, this restriction, which was made for technical simplicity, can be omitted. We then have to modify the boundary coefficient $C_{R}\left(\omega^{h}\right)$ in order to take into account only the vortices which lie in $\Omega_{R}$, and our proof can be easily adapted to this situation.

It remains now to consider extensions of this method, such as the 3-dimensional case and methods using more accurate numerical boundary conditions on $\Gamma_{R}$.

Acknowledgments. The author wishes to thank S. Gallic and P. A. Raviart for their kind help during the preparation and writing of this paper.

Centre de Mathématiques Appliquées

Ecole Polytechnique

Unité de Recherche Associée au CNRS-756

91128 Palaiseau Cédex, France

1. J. T. Beale \& A. Majda, "Vortex methods. II: Higher order accuracy in two and three dimensions," Math. Comp., v. 39, 1982, pp. 29-52.

2. P. G. Ciariet \& P. A. Raviart, "Maximum principle and uniform convergence for the finite element method," Comput. Methods Appl. Mech. Engrg., v. 2, 1973, pp. 17-31.

3. G. H. Cottet, Thèse de 3ème cycle, Université Paris VI, 1982.

4. G. H. COTtET, "A new approach for the analysis of vortex methods in two and three dimensions," Ann. Inst. H. Poincaré. (Submitted.)

5. G. H. Cottet \& P. A. Raviart, "On Particle in Cell Methods for the Vlasov-Poisson equations," Transport Theory Statist. Phys., v. 15, 1986, pp. 1-31.

6. O. Hald, "The convergence of a vortex method. II," SIAM J. Numer. Anal., v. 16, 1979, pp. $726-755$

7. R. W. Hockney \& J. W. Eastwood, Computer Simulation Using Particles, McGraw-Hill, New York, 1981.

8. A. Leonard, “Vortex methods for flow simulations,” J. Comput. Phys., v. 37, 1980, pp. 289-335.

9. R. RANNACHER \& R. SCOTT, "Some optimal error estimates for piecewise linear finite element approximations," Math. Comp., v. 38, 1982, pp. 437-446.

10. P. A. Raviart, An Analysis of Particle Methods, CIME Course, COMO, 1983.

11. V. THOMEE, "Discrete interior Schauder estimates for elliptic difference operators," SIAM J. Numer. Anal., v. 5, 1968, pp. 626-645. 